Lichens are by definition symbiotic organisms composed of a fungal partner, the mycobiont ISSN Online 0974-7907 Print 0974-7893 and one or more photosynthetic partners, the photobiont, that may be either a green alga or

OPEN ACCESS a cyanobacterium. Lichens are regarded as an example of controlled parasitism, because the fungus seems to obtain most of the benefits and the photobiont may grow more slowly in the lichenized state than when free-living (Ahmadjian 1993).

They dominate other groups of organisms in as much as $8 \%$ of the earth's surface (Ahmadjian 1993, 1995). The associated entity grows at an average rate of 1-5 mm per year and persists for tens or hundreds of years on their substratum. In the tropics and subtropics some rapidly growing lichens even colonize the surface of leaves as epiphylls.

The growth forms of lichens are usually conspicuous and among the terrestrial autotrophs of the world, lichens exhibit intriguing variation in miniature. They are categorized primarily based on their morphology and size into three major types, viz., crustose (crust like), foliose (leaf like) and fruticose (shrubby). The lichens belonging to the former category are called microlichens and the latter two are referred to as macrolichens. They colonize a great variety of substrates such as rocks, soil, humus, wood substrates as tree trunks, branches and logs, animal shells, bones, insect backs, synthetic materials as plastic taps and substrates derived from mineral sources such as bricks, cement, concrete roofs and walls and glass and iron, amongst others (Brightman \& Seaward 1978; Hale

\section{Lichens of the Mahabaleshwar Panchgani ECOSENSITIVE ZONE (MPESZ), MAHARASHTRA, INDIA}

\section{Gargee S. Pandit}

Agharkar Research Institute, G.G. Agarkar Road, Pune, Maharashtra 411004, India gargee.pandit @gmail.com

\section{3; Sipman 1994; Schroeter \& Sancho 1996).}

Many of the lichen species have proved economically very beneficial and continue to hold significant commercial implications particularly in cosmetic and perfumery industries. A large number of chemicals called lichen substances unique to lichens have made them useful as a source of dyes, medicines, agrochemicals and other exploitable compounds. They play an important role in the mineral cycling patterns of their ecosystem, particularly if cyanolichens are the dominant components.

The Western Ghats have attracted the attention of naturalists for nearly a century in course of the studies on the biological material of their interests or in their discussions on the biogeography of the Indian biota. The complex topography of the Western Ghats with a wide range of microclimatic and soil conditions have resulted in a mosaic of plant communities and animal associations unique to itself. Besides being biologically rich in genera and species the Western Ghats is rich in endemics too. Many new and endemic lichen species have also been reported from this region (Makhija et al. 2004; Dube et al. 2005; Makhija et al. 2005; Makhija et al. 2006; Chitale \& Makhija 2008; Chitale et al. 2008; Chitale et al. 2009;

DOI: http://dx.doi.org/10.11609/JoTT.03784.5784-91

Editor: Aparna Watve, Pune, India.

Date of publication: 26 May 2014 (online \& print)

Manuscript details: Ms \# 03784 | Received 24 September 2013 | Final received 31 January 2014 | Finally accepted 01 May 2014

Citation: Pandit, G.S. (2014). Lichens of the Mahabaleshwar Panchgani Ecosensitive zone (MPESZ), Maharashtra, India. Journal of Threatened Taxa 6(5): 5784-5791; http://dx.doi.org/10.11609/JoTT.03784.5784-91

Copyright: ( ) Pandit 2014. Creative Commons Attribution 4.0 International License. JoTT allows unrestricted use of this article in any medium, reproduction and distribution by providing adequate credit to the authors and the source of publication.

Funding: The Department of Science and Technology-SERB, SB/FT/LS-187/2012, New Delhi.

Competing Interest: The authors declare no competing interests.

Acknowledgements: The author is grateful to the Director, Agharkar Research Institute Pune, for the laboratory facilities. Thanks are also due to Dr. Aparna Watve for her inspiration in making the project and as one of the valuable reviewer of this paper; and my guide Dr. Urmila Makhija who made me a lichenologist and also Dr. Bharati Sharma who has directly and indirectly helped me in the work, and Mr. Subhash Gaikwad for his technical assistance and suggestions. I would also like to thank the Bombay Environmental Action Group (BEAG) for providing the map required by me for the study area. 
Makhija et al. 2009; Dube \& Makhija 2010; Singh \& Sinha 2010; Bajpai \& Upreti 2011; Chitale et al. 2011; Bajpai et al. 2012;). Lateritic plateaus and basalt outcrops are a special habitat seen in the northern Western Ghats. They are known for high endemism and dominance of certain function groups such as carnivorous plants (Watve 2013). They are seen at altitude above $900 \mathrm{~m}$ in the Western Ghats.

During the previous surveys undertaken for documentation of lichen diversity of the region, rocky plateaus of laterite as well as basalt were seen to have abundance of saxicolous lichens. Though the saxicolous lichen species of India have been studied by Indian and foreign lichenologists, they are available in the form of scattered publications and all are dealing mainly with the taxonomy (Awasthi 1965, 1988, 1991, 2000; Schubert \& Klement 1966; Patwardhan \& Badhe 1972; Degelius 1974).

In view of this, the present paper documents lichens of Mahabaleshwar-Panchgani Ecosensitive Zone (MPESZ) in the northern Western Ghats, and reports the diverse lichen forms and their preferred substrates.

Study area: MPESZ lies roughly between $17^{\circ} 55^{\prime} \mathrm{N}-73^{\circ} 40^{\prime} \mathrm{E} \& 17.00^{\circ} \mathrm{N}-73.82^{\circ} \mathrm{E}$. The lateritic plateaus, better known as the tablelands, have been studied by many geologists and geographers
(Widdowson \& Cox 1996; Widdowson 1997; Ollier \& Sheth 2008). The area was a famous hill station since the British period and botanically very well known due to the work of Blatter (1909), Razi (1952), Puri \& Mahajan (1960), Deshpande et al. (1993, 1995). However, the focus of these studies was on angiosperms. Old records of lichens of the region are available, but needed to be updated. New records of lichens from Mahabaleshwar and Koyna region have been recently published (Bajpai \& Upreti 2011; Bajpai et al. 2012). Lichenology group from Agharkar Research Institute has consistently reported many lichens from this region (Dube et al. 2005; Chitale 2007; Chitale et al. 2008; Chitale \& Makhija 2008; Chitale et al. 2009; Dube \& Makhija 2010; Chitale et al. 2011). However, this is the first ever checklist of the lichens of this region, and describes the prevalent forms with their microhabitat needs.

MPESZ area is dominated by semi-evergreen forests of Memecylon-Syzygium-Actinodaphne series as described by Pascal (1988). Much of the hill slopes are biotically modified and dominated by Catunaregam spinosa, Scutia myrtina and Carissa congesta thickets. Herbaceous vegetation dominates open areas in the monsoon period (June-September). Five tablelands are located within the Panchgani Municipal Corporation limits, and another fourteen plateaus are reported

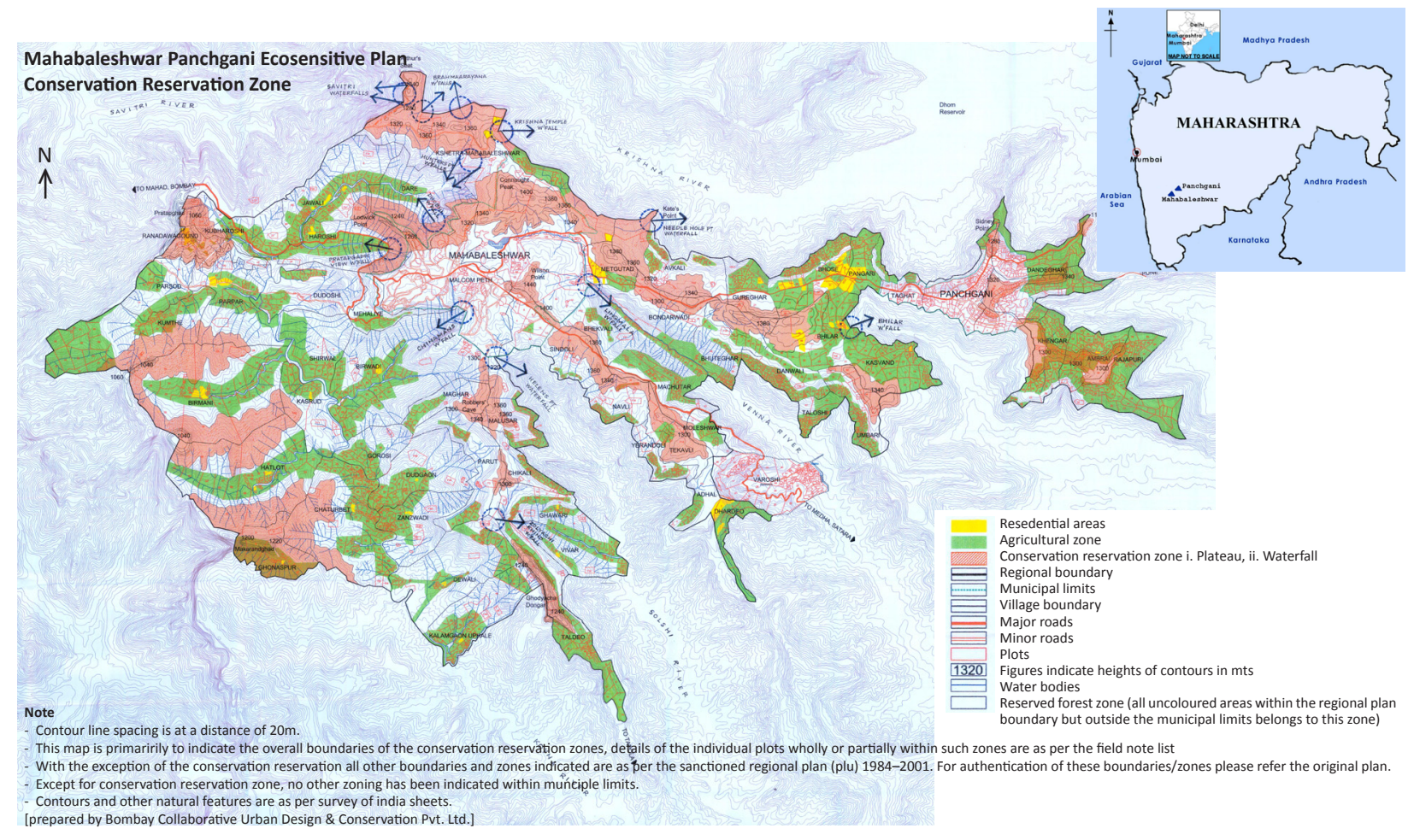

Figure 1. Study area. (C) The Bombay Environmental Action Group (BEAG) 


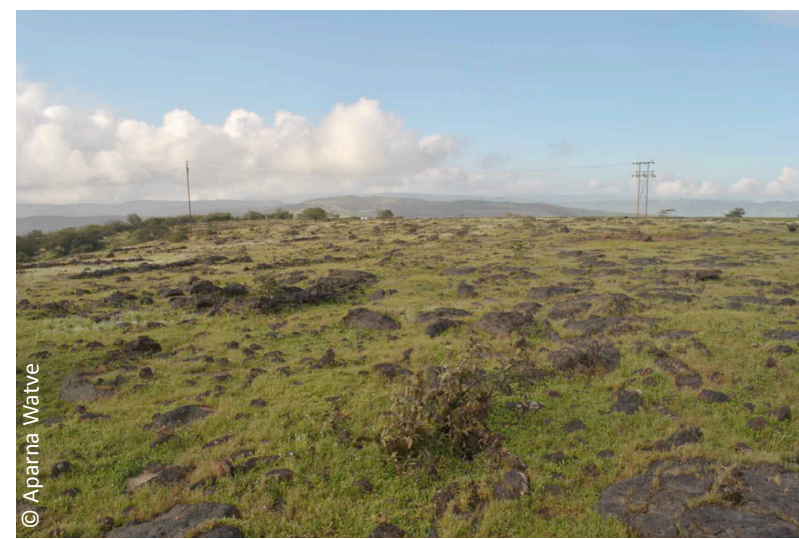

Image 1. Wilson Point

from Mahabaleshwar proper. Notable amongst these is the Wilson Point (Image 1) with an altitude of about 1000-1400 m, which is the highest point in the region. All these plateaus are declared conservation zones as per the development plan of the region and tablelands of Panchgani are natural heritage sites (Image 2). Tetali et al. (2000) and Mishra \& Singh (2001) have described more than 20 endemic and threatened flowering plants from this region.

Methods: Primary data on MPESZ lichens was collected during 2001-2010 as a part of surveys for Maharashtra lichens and studies on microlichens (Chitale 2007). The floristic surveys covered dry as well as wet seasons, diverse habitats (forests, scrublands and rocky plateaus). Lichen specimens were collected using standard field survey techniques and reference specimens are deposited in Agharkar Research Institute lichen repository (Ajrekar Mycological Herbarium $\mathrm{AMH}$ ). Ecological notes regarding substrate, forms, abundance were recorded on field.

All the specimens collected were studied for their morphology, anatomy and chemistry and identified at Agharkar Research Institute (ARI) using the most recent literature available on lichen taxonomy.

Secondary data was collected from previously published literature from the study area (listed above) which was mostly about new records and lichen descriptions. Earlier lichen collections from ARI lichen repository were also scanned to make a complete regional checklist of species reported so far.

Results and Discussion: A list of species of lichens reported from MPESZ is given Table 1 . They include, 25 families represented by 43 genera and about 129 species which is $5.6 \%$ of the total lichens known from India. Of these, 110 species are exclusively corticolous (bark or twig dwelling), 20 species are saxicolous (rock

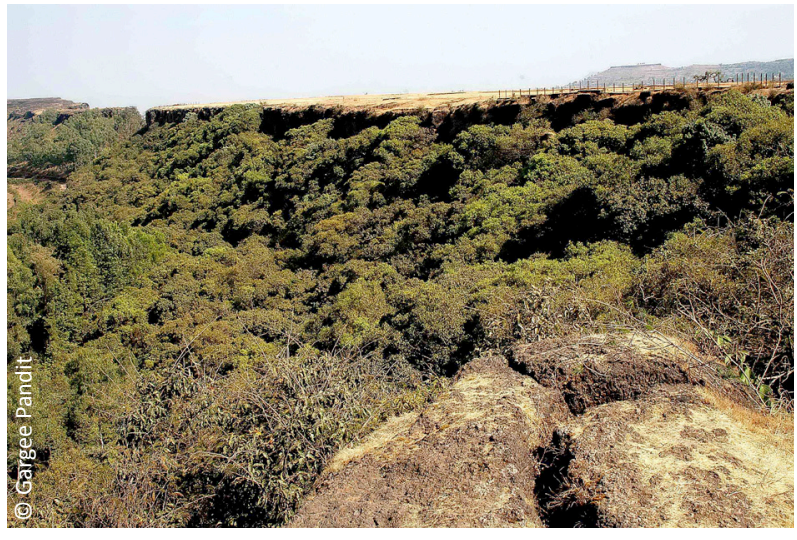

Image 2. Table Land-Panchgani

dwelling), three species are muscicolous and only one species is exclusively terricolous. Lichens can also be categorized as macrolichens, including, foliose lichens (49 spp.); fruticose (3 spp.); squamulose (4 spp.), leprose (3 spp.) and microlichens i.e. crustose lichens (72 spp.).

So far only five lichen species have been recorded from the Panchgani Plateau but the number is likely to increase as currently exploratory, ecological studies have been started as part of DST-Fast-track Scheme. Some species probably form their origin here, e.g., Diorygma megasporum Kalb, Staiger \& Elix, Diorygma panchganiense Makhija, Chitale \& B.O. Sharma. (Makhija et al. 2009). Observations also show that excessive trampling and disturbance has eroded lichen flora from the main parts of Panchgani tableland, while the peripheral part of the plateau has a better preserved lichen crust. Therefore, it is necessary to study and then plan protection measures to protect this unique lichen diversity of these unique plateaus.

Schubert \& Klement (1966) in their study tour to northern and central India have collected several lichens from these areas of which 16 species are recorded from Maharashtra. Degelius (1974) in his monograph on the lichen genus Collema have reported five saxicolous species from Maharashtra (Satara District, near Panchgani). Three species of the genus Rhizocarpon have so far been recorded from Maharashtra-Rhizocarpon concentricum (Davies) Beltram, Rhizocarpon distinctum Th. Fr., Rhizocarpon obscuratum (Ach.) Massal. (Singh, 1980)-but the material on which the record of earlier known species are based was not available for study. Chitale Gayatri and Dube Archana during 2001-2007 have taxonomically explored the state of Maharashtra and also the MPESZ area for their doctoral studies and have published new species (Chitale 2007; Dube 2007). Unfortunately Rhizocarpon genus was not recollected. 
Table 1. List of lichen species recorded in MPESZ with description of growth form and substrate

\begin{tabular}{|c|c|c|c|c|}
\hline & Taxa & Family & Growth forms & Substrate \\
\hline 1 & Anisomeridium albisedum (Nyl.) R. C. Harris & Monoblastiaceae & Crustose & Corticolous \\
\hline 2 & Arthothelium albescens Patw. \& Makhija & Arthoniaceae & Crustose & Corticolous \\
\hline 3 & Arthothelium nigrodiscum Patw. \& Makhija & Arthoniaceae & Crustose & Corticolous \\
\hline 4 & Arthothelium subruanum Makhija \& Patw. & Arthoniaceae & Crustose & Corticolous \\
\hline 5 & Aspicilia calcarea (L.) Sommerf. & Megasporaceae & Crustose & Saxicolous \\
\hline 6 & Bacidia alutacea (Kremp.) Zahlbr. & Ramalinaceae & Crustose & Corticolous \\
\hline 7 & Bacidia fusconigrescens (Kremp.) Zahlbr. & Ramalinaceae & Crustose & Corticolous \\
\hline 8 & Bacidia personata Malme & Ramalinaceae & Crustose & Corticolous \\
\hline 9 & Bacidia rubella (Hoffm.) Massal. & Ramalinaceae & Crustose & Corticolous \\
\hline 10 & Buellia panchganiensis Makhija \& Dube (Table Land, Panchgani) & Caliceaceae & Crustose & Saxicolous \\
\hline 11 & Buellia tabularis Makhija \& Dube (Table Land, Panchgani) & Caliceaceae & Crustose & Saxicolous \\
\hline 12 & Caloplaca abuenis Joshi \& Upreti & Teloschistaceae & Crustose & Saxicolous \\
\hline 13 & Caloplaca amarkantakana Joshi \& Upreti & Teloschistaceae & Crustose & Saxicolous \\
\hline 14 & Caloplaca cupulifera (Vain.) Zahlbr. & Teloschistaceae & Crustose & Saxicolous \\
\hline 15 & Caloplaca flavorubescens (Huds.) J.R.Laundon (Table Land, Panchgani) & Teloschistaceae & Crustose & Corticolous \\
\hline 16 & Caloplaca pollinii (A.Massal.) Jatta & Teloschistaceae & Crustose & Corticolous \\
\hline 17 & Cladonia scabriuscula (Delise) Nyl. & Cladoniaceae & Fruticose & Corticolous \\
\hline 18 & Collema conglomeratum Hoffm. Var. crassiusculum (Malme) Degel. & Collemataceae & Foliose & Corticolous \\
\hline 19 & Collema furfureolum Mull. Arg. & Collemataceae & Foliose & Saxicolous \\
\hline 20 & Collema leptaleum Tuck. var. biliosum (Mont.) Degel. & Collemataceae & Foliose & Corticolous \\
\hline 21 & Collema polycarpon Hoffm. var. polycarpon & Collemataceae & Foliose & Saxicolous \\
\hline 22 & Collema pulcellum Ach. var. subnigrescens (Mull. Arg.) Degel. & Collemataceae & Foliose & Corticolous \\
\hline 23 & Collema tenax var. tenax (Sw.) Ach. & Collemataceae & Foliose & Saxicolous \\
\hline 24 & Cryptothecia lunulata (Zahlbr.) Makhija \& Patw. & Arthoniaceae & Crustose & Corticolous \\
\hline 25 & Diorygma "microsporum" ad int. & Graphidaceae & Crustose & Corticolous \\
\hline 26 & Diorygma "patwardhanii" ad int. & Graphidaceae & Crustose & Corticolous \\
\hline 27 & Diorygma albocinerascens Makhija, Chitale \& B.O. Sharma & Graphidaceae & Crustose & Corticolous \\
\hline 28 & Diorygma albovirescens Makhija, Chitale \& B.O. Sharma & Graphidaceae & Crustose & Corticolous \\
\hline 29 & Diorygma excipuloconvergentum Makhija, Chitale \& B.O. Sharma & Graphidaceae & Crustose & Corticolous \\
\hline 30 & Diorygma junghuhnii (Mont. \& Bosch) Kalb. in Kalb & Graphidaceae & Crustose & Corticolous \\
\hline 31 & Diorygma megaspermum Makhija, Chitale \& B.O. Sharma & Graphidaceae & Crustose & Corticolous \\
\hline 32 & Diorygma megasporum Kalb, Staiger \& Elix & Graphidaceae & Crustose & Corticolous \\
\hline 33 & Diorygma megistosporum Makhija, Chitale \& B.O. Sharma & Graphidaceae & Crustose & Corticolous \\
\hline 34 & Diorygma panchganiense Makhija, Chitale \& B.O. Sharma & Graphidaceae & Crustose & Corticolous \\
\hline 35 & Diploschistes cf. rampoddensis (Nyl.) Zahlbr. (Table Land, Pgani) & Thelotremataceae & Crustose & Saxicolous \\
\hline 36 & Endocarpon subrosettum A. Singh \& Upreti & Verrucariaceae & Crustose & Saxicolous \\
\hline 37 & Fissurina cingalina (Nyl.) Staiger & Graphidaceae & Crustose & Corticolous \\
\hline 38 & Graphis duplicata Ach. & Graphidaceae & Crustose & Corticolous \\
\hline 39 & Graphis lineola Ach. & Graphidaceae & Crustose & Corticolous \\
\hline 40 & Graphis nigroglauca Leight. & Graphidaceae & Crustose & Corticolous \\
\hline 41 & Graphis parilis Kremph. & Graphidaceae & Crustose & Corticolous \\
\hline 42 & Graphis platycarpa Eschw. & Graphidaceae & Crustose & Corticolous \\
\hline 43 & Graphis polystriata Makhija, A. Dube, Adaw. \& Chitale & Graphidaceae & Crustose & Corticolous \\
\hline 44 & Graphis proserpens Vain. & Graphidaceae & Crustose & Corticolous \\
\hline
\end{tabular}




\begin{tabular}{|c|c|c|c|c|}
\hline & Taxa & Family & Growth forms & Substrate \\
\hline 45 & Graphis sp. 1 & Graphidaceae & Crustose & Corticolous \\
\hline 46 & Graphis subserpentina (Nyl.) Mull Arg. & Graphidaceae & Crustose & Corticolous \\
\hline 47 & Graphis treblocarpa (Bel.) Nyl. & Graphidaceae & Crustose & Corticolous \\
\hline 48 & Graphis tsunodae Zahlbr. & Graphidaceae & Crustose & Corticolous \\
\hline 49 & Hemithecium nakanishianum (Patw. \& C.R. Kulkarni) Makhija \& Dube & Graphidaceae & Crustose & Corticolous \\
\hline 50 & Hemithecium norsticticum Makhija \& Dube & Graphidaceae & Crustose & Corticolous \\
\hline 51 & Hemithecium pyrrhochroa (Mont. \& Bosch.) V. Tewari \& Upreti & Graphidaceae & Crustose & Corticolous \\
\hline 52 & Heterodermia albicans (Pers.) Swinscow \& Krog L & Physciaceae & Foliose & Corticolous \\
\hline 53 & Heterodermia angustiloba (Miill. Arg.) Awasthi & Physciaceae & Foliose & Corticolous \\
\hline 54 & Heterodermia boryii (Fee) K.P. Singh \& S.R. Singh & Physciaceae & Foliose & Corticolous \\
\hline 55 & Heterodermia diademata (Taylor) Awasthi & Physciaceae & Foliose & Corticolous \\
\hline 56 & Heterodermia hypocaesia (Yesuda D.D. Awasthi & Physciaceae & Foliose & Corticolous \\
\hline 57 & Heterodermia incana (Stirton) Zahlbr. & Physciaceae & Foliose & Corticolous \\
\hline 58 & Heterodermia japonica (Sato) Swinscow \& Krog & Physciaceae & Foliose & Corticolous \\
\hline 59 & Heterodermia leucomelos (L.) Poelt & Physciaceae & Foliose & Corticolous \\
\hline 60 & Heterodermia podocarpa (Bel.) Awasthi & Physciaceae & Foliose & Corticolous \\
\hline 61 & Heterodermia pseudospeciosa (Kurok.) W. Culb. & Physciaceae & Foliose & Corticolous \\
\hline 62 & Heterodermia sp. & Physciaceae & Foliose & Corticolous \\
\hline 63 & Heterodermia speciosa (Wulfen) Trevisan & Physciaceae & Foliose & Corticolous \\
\hline 64 & Hypotrachyna awasthi Hale \& Patw. & Parmeliaceae & Foliose & Corticolous \\
\hline 65 & Lecanora alba Lumbsch & Lecanoraceae & Crustose & Corticolous \\
\hline 66 & Lecanora allophana (Ach.) Röhl. & Lecanoraceae & Crustose & Corticolous \\
\hline 67 & Lecanora andina Rasanen & Lecanoraceae & Crustose & Corticolous \\
\hline 68 & Lecanora austrointumescens Lumbsch \& Elix & Lecanoraceae & Crustose & Corticolous \\
\hline 69 & Lecanora cenisia Ach. & Lecanoraceae & Crustose & Corticolous \\
\hline 70 & Lecanora cf. imshaugii Brodo & Lecanoraceae & Crustose & Corticolous \\
\hline 71 & Lecanora chlarotera Nyl. & Lecanoraceae & Crustose & Corticolous \\
\hline 72 & Lecanora expallens Ach. & Lecanoraceae & Crustose & Corticolous \\
\hline 73 & Lecanora interjecta Mull. Arg. & Lecanoraceae & Crustose & Corticolous \\
\hline 74 & Lecanora lavidofusca Mull. Arg. & Lecanoraceae & Crustose & Corticolous \\
\hline 75 & Lecanora sp. 1 (Table Land, Pgani) & Lecanoraceae & Crustose & Corticolous \\
\hline 76 & Lepraria coriensis (Hue) Sipman & Stereocaulaceae & Leprose & Corticolous \\
\hline 77 & Lepraria lobificans Nyl. & Stereocaulaceae & Leprose & Saxicolous \\
\hline 78 & Lepraria sp. & Stereocaulaceae & Leprose & Corticolous \\
\hline 79 & Leptogium azureum (Sw.) Mont. & Collemataceae & Foliose & $\begin{array}{c}\text { Corticolous or } \\
\text { Saxicolous }\end{array}$ \\
\hline 80 & Leptogium burnetiae C.W. Dodge var. hirsutum (Sierk) P.M. Jorg. & Collemataceae & Foliose & $\begin{array}{c}\text { Corticolous/ } \\
\text { Muscicolous/ } \\
\text { Saxicolous }\end{array}$ \\
\hline 81 & Leptogium chloromelum (Sw.) Nyl. & Collemataceae & Foliose & Corticolous \\
\hline 82 & Leptogium cochleatum (Dicks.) P.M. Jorg. \& P. James & Collemataceae & Foliose & Corticolous \\
\hline 83 & Leptogium cyanescens (Ach.) Korb. & Collemataceae & Foliose & $\begin{array}{l}\text { Corticolous/ } \\
\text { Muscicolous }\end{array}$ \\
\hline 84 & Leptogium denticulatum Nyl. & Collemataceae & Foliose & $\begin{array}{l}\text { Corticolous/ } \\
\text { Muscicolous }\end{array}$ \\
\hline 85 & Leptogium gelatinosum (With) J.R. Laundon & Collemataceae & Foliose & Corticolous \\
\hline 86 & Leptogium indicum D.D. Awasthi \& Akhtar & Collemataceae & Foliose & Corticolous \\
\hline 87 & Leptogium javanicum Mont. & Collemataceae & Foliose & $\begin{array}{l}\text { Corticolous/ } \\
\text { Saxicolous }\end{array}$ \\
\hline
\end{tabular}




\begin{tabular}{|c|c|c|c|c|}
\hline & Taxa & Family & Growth forms & Substrate \\
\hline 88 & Leptogium phyllocarpum (Pers.) Mont. & Collemataceae & Foliose & Corticolous \\
\hline 89 & Leptogium propaguliferum Vain. & Collemataceae & Foliose & Corticolous \\
\hline 90 & Leptogium subazureum Dube \& Makhija & Collemataceae & Foliose & Corticolous \\
\hline 91 & Leptogium ulvaceum (Pers.) Vain & Collemataceae & Foliose & Terricolous \\
\hline 92 & Lopezaria isidiza (Makhija \& Nagarkar) Aptroot \& Sipman & Ramalinaceae & Crustose & Corticolous \\
\hline 93 & Micarea sp. & Pilocarpaceae & Crustose & Corticolous \\
\hline 94 & Mycomicrothelia hemispherica (Mull. Arg.) D. Hawksw. & Arthopyreniaceae & Crustose & Corticolous \\
\hline 95 & Myelochroa aurulenta (Tuck.) Elix \& Hale & Parmeliaceae & Foliose & Corticolous \\
\hline 96 & Pallidogramme commutabilis (Kremp.) Chitale \& Makhija & Graphidaceae & Crustose & Corticolous \\
\hline 97 & Parmeliella brisbanensis (Knight.) P.M. Jorg. \& D.J. Galloway & Pannariaceae & $\begin{array}{c}\text { Crustose-squamulose } \\
\text { to foliose }\end{array}$ & Corticolous \\
\hline 98 & Parmelinella simplicior (Hale) Elix \& Hale & Parmeliaceae & Foliose & Corticolous \\
\hline 99 & Parmelinella wallichiana (Tayl.) Elix \& Hale & Parmeliaceae & Foliose & Corticolous \\
\hline 100 & Parmotrema sanctiangelii (Lynge) Hale & Parmeliaceae & Foliose & Corticolous \\
\hline 101 & Parmotrema tinctorum (Nyl.) Hale & Parmeliaceae & Foliose & $\begin{array}{c}\text { Corticolous/ } \\
\text { Saxicolous } \\
\end{array}$ \\
\hline 102 & Pertusaria alutacea (Kremph.) Zahlbr. & Pertusariaceae & Crustose & Corticolous \\
\hline 103 & Pertusaria cf. depressa (Fee) Mont. Et Bosch & Pertusariaceae & Crustose & Corticolous \\
\hline 104 & Pertusaria corallina (L.) Arnold & Pertusariaceae & Crustose & Corticolous \\
\hline 105 & Pertusaria pertusa (L.) Tuck. & Pertusariaceae & Crustose & Corticolous \\
\hline 106 & Pertusaria quassiae (Fée) Nyl. & Pertusariaceae & Crustose & Corticolous \\
\hline 107 & Phaeophyscia endococcina var. endococcinoides (Poelt) Essl. & Physciaceae & Foliose & Corticolous \\
\hline 108 & Phaeophyscia hispidula (Ach.) Moberg & Physciaceae & Foliose & Corticolous \\
\hline 109 & Phaeophyscia pyrrophora (Poelt) Awasthi \& Joshi & Physciaceae & Foliose & Corticolous \\
\hline 110 & Phlyctis karnatakana S. Joshi \& Upreti & Phlyctidaceae & Crustose & Corticolous \\
\hline 111 & Phyllopsora corallina (Eschw.) Mull. Arg. & Biatoraceae & Squamulose, & Corticolous \\
\hline 112 & Physcia abuensis D.D. Awasthi \& S.R. Singh & Physciaceae & Foliose & Corticolous \\
\hline 113 & Physcia integrata Nyl. & Physciaceae & Foliose & Corticolous \\
\hline 114 & Physcia tribacoides Nyl. & Physciaceae & Foliose & Corticolous \\
\hline 115 & Physcia undulata Moberg & Physciaceae & Foliose & Corticolous \\
\hline 116 & Porina sp. & Porinaceae & Crustose & Corticolous \\
\hline 117 & Pyrenopsis sp. & Lichinaceae & & \\
\hline 118 & Pyxine cocoes var. cocoes (Swartz) Nyl. & Physciaceae & Foliose & Corticolous \\
\hline 119 & Pyxine cocoes var. prominula (Stirt.) D.D. Awasthi & Physciaceae & Foliose & Corticolous \\
\hline 120 & Pyxine petricola var. petricola Nyl. & Physciaceae & Foliose & Corticolous \\
\hline 121 & Remototrachyna awasthi (Hale \& Patw.) Divakar \& Crespo & Parmeliaceae & Foliose & Corticolous \\
\hline 122 & Rimelia reticulata (Taylor) Hale \& A. Fletcher & Parmeliaceae & Foliose & Corticolous \\
\hline 123 & Staurothele clopima (Wahlenb.) Th. Fr. & Verrucariaceae & $\begin{array}{c}\text { Crustose } \\
\text {-squamulose }\end{array}$ & Saxicolous \\
\hline 124 & Staurothele fissa (Taylor) Zack. & Verrucariaceae & Crustose-squamulose & Saxicolous \\
\hline 125 & Thelotrema monosporum Nyl. & $\begin{array}{l}\text { Thelotremoid } \\
\text {-Graphidaceae }\end{array}$ & Crustose & Corticolous \\
\hline 126 & Trapelia placiodiodes Coppins \& James & Tapelariaceae & Crustose & Saxicolous \\
\hline 127 & Usnea complanata (Mull. Arg.) Motyka & Parmeliaceae & Fruticose & Corticolous \\
\hline 128 & Usnea ghattensis G. Awasthi & Parmeliaceae & Fruticose & Corticolous \\
\hline 129 & Verrucaria acrotella Ach. & Verrucariaceae & Crustose & Saxicolous \\
\hline
\end{tabular}




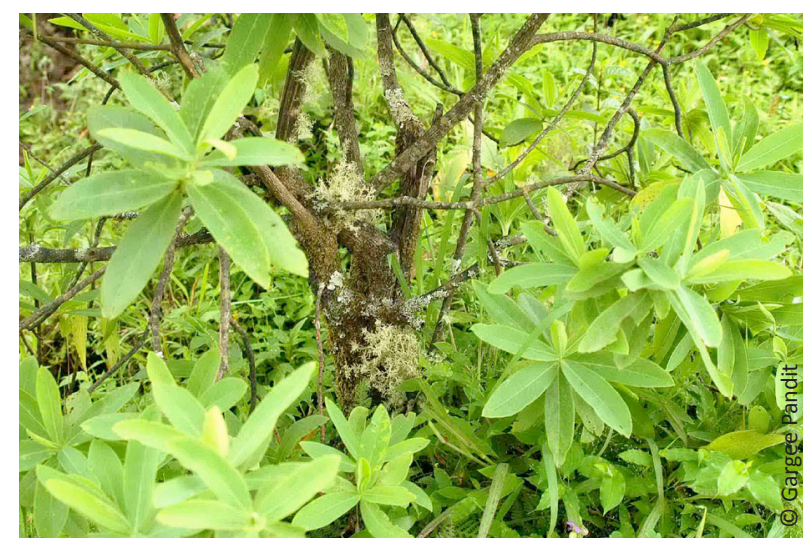

Image 3. Usnea ghattensis

But there is a hope of finding it on these outcrops with thorough explorations, as they are saxicolous species (rock dwelling).

Many of these species are likely to be endemic to the special habitats in this region. They are most vulnerable to extinction as they occur in narrow geographical areas and it is extremely important to document the existing vegetation and study the effect of biotic pressures on it.

Natural habitats in MPESZ are gradually degrading due to increasing human pressures. The protection offered by ecosensitive zone category or natural heritage tag or conservation zone declaration has slowed down the habitat depletion caused by land-use change and rapid urbanization. However, increasing pressure for fuelwood, grazing, insensitive tourism are serious threats to natural biodiversity. Lichen flora, is sensitive to even mild disturbance of their habitats and hence needs special protection. The role of lichens as pioneer species in a habitat, as indicator taxa and complex biological symbionts needs to be emphasized. It is urgently necessary that steps are taken to halt the process and thereby conserve the diversity of species characteristic and endemic to these areas. It is hoped that this review of MPESZ lichens attracts attention to this lesser-known but ecologically significant group of organisms and appropriate conservation measures are urgently taken. However, increasing pressure for fuelwood, grazing, insensitive tourism are serious threats to natural biodiversity (Image 3).

\section{References}

Ahmadjian, V. (1993). The lichen symbiosis, John Wiley \& Sons, USA Inc. pp 1-250.

Ahmadjian, V. (1995). Lichens are more important than you think. Bioscience 45: 124.

Awasthi, D.D. (1965). Catalogue of the lichens from India, Nepal,
Pakistan and Ceylon. Beihefte zur Nova Hedwigia 17: 1-137.

Awasthi, D.D. (1988). A key to the macrolichens of India and Nepal. Journal of Hattori Botanical Laboratory 65: 07-302.

Awasthi, D.D. (1991). A key to the microlichens of India, Nepal and Sri Lanka. Bibliotheca Lichenologica 40: 1-337.

Awasthi, D.D. (2000). Lichenology in Indian subcontinent, Bishen Singh Mahendra Pal Singh, Dehra Dun, India, 145pp.

Blatter, (1909). The flora of Panchgani. Journal of the Bombay Natural History Society 19: 314-332.

Brightman, S. \& M.R.D. Seaward (1978). Lichens on man made substrates, pp. 253-293. In: Seaward M.R.D (ed.). Lichen Ecology. Academic Press.

Bajpai, R. \& D.K. Upreti (2011). New records of lichens from Mahabaleshwar and Koyna areas of Satara District, Maharashtra, India. Geophytology 40(1\&2): 61-68.

Bajpai, R., D.K. Upreti, S. Nayaka \& U. Dubey (2012). Lichen flora in and around Mahabaleshwar, Satara District, Maharashtra with Lecanora expallens Ach. as new record to Indian lichen flora. Phytotaxonomy 12: $123-130$.

Chitale, G.S. (2007). Studies on the Microlichens of Maharashtra. PhD Thesis. Submitted to University of Pune, 304pp.

Chitale, G., A. Dube \& U. Makhija (2008). The lichen genus Physcia and allied genera from Maharashtra, India. Geophytology 37: 13-21.

Chitale, G. \& U. Makhija (2008). A new species of the lichen genus Brigantiaea from India. Mycotaxon 104: 409-413.

Chitale, G., U. Makhija \& B. Sharma (2009). New combinations and new species in the lichen genera Hemithecium and Pallidogramme. Mycotaxon 108: 83-92.

Chitale, G., U. Makhija \& B. Sharma (2011). Additional species of Graphis from Maharashtra, India. Mycotaxon 115: 469-480; http:// dx.doi.org/10.5248/115.469

Degelius, G. (1974). The lichen genus Collema with special reference to the Extra-European species. Symbolae Botanicae Upsalienses 20: $1-215$.

Deshpande, S., B.D. Sharma \& M.P. Nayar (1993). Flora of Mahabaleshwar and Adjoining Areas, Maharashtra. Flora of India Series 3 - Vol. 1. Botanical Survey of India, Calcutta, 431pp.

Deshpande, S., B.D. Sharma \& M.P. Nayar (1995). Flora of Mahabaleshwar and Adjoining Areas, Maharashtra. Flora of India Series 3 - Vol. 2. Botanical Survey of India, Calcutta, 433-776pp.

Pascal, J.P. (1988). Wet Evergreen Forests of the Western Ghats of India. Institute Francais de Pondicherry, Pondicherry.

Dube, A. (2007). A contribution to our knowledge of the macrolichens of Maharashtra. PhD Thesis. Submitted to the University of Pune, 1-150pp.

Dube, A., G. Chitale \& U. Makhija (2005). The lichen genera Dirinaria and Pyxine (Family: Physciaceae) from Maharashtra, India. Phytotaxonomy 5: 83-89.

Dube, A. \& U. Makhija (2010). Occurence of four additional non-hairy species of Leptogium from Maharashtra, India. Lichenologist 42(6): 701-710; http://dx.doi.org/10.1017/S0024282910000332

Hale, M.E. (1983). The Biology of lichens - $3^{\text {rd }}$ Edition. Edward Arnold (Australia) Pvt. Ltd., Australia, 180pp.

Makhija, U., G. Chitale \& A. Dube (2006). An account of the lichen genus Lecanora from Maharashtra. (Communicated - Ministry of Environment \& Forests publication).

Makhija, U., G. Chitale \& B. Sharma (2009). New species and new records of Diorygma (Graphidaceae) from India: species with convergent exciples. Mycotaxon 109: 379-392.

Makhija, U., G. Chitale \& A. Dube (2004). The lichen genus Heterodermia (Family: Physciaceae) from Maharashtra. Geophytology 34: 43-55.

Makhija, U., A. Dube, B. Adawadkar \& G. Chitale (2005). Five transseptate species of Hemithecium from India. Mycotaxon 93: 365372.

Makhija, U., A. Dube, B. Adawadkar \& G. Chitale (2006). Some species of lichen genera Dyplolabia and Graphis from Maharashtra, India. Geophytology 36(1\&2): 61-68.

Mishra, D.K. \& N.P. Singh (2001). Endemic and Threatened Flowering Plants of Maharashtra. Botanical Survey of India, Calcutta, 414pp. 
Ollier, C. \& H.C. Sheth (2008). The High deccan duricrusts of India and their significance for the laterite issue. Journal of Earth System Science, 117: 537-551.

Patwardhan, P.G. \& P.D. Badhe (1972). Contributions to the lichen flora of western India - IV. Journal of Shivaji University 5(10): 135-139.

Puri, G.S. \& S.D. Mahajan (1960). The study of the evergreen vegetation of Mahabaleshwar area. Bulletin of Botanical Survey of India 2: 109-137.

Razi, B.A. (1952). Flora of Panchgani. DSc Thesis. University of Poona, Poona.

Schroeter, B. \& L.G. Sancho (1996). Lichens growing on glass in Antarctica Lichenologist 28: 385-390.

Schubert, R. \& O. Klement (1966). Beitrag zur Flechten-Flora von Nord-und Mittelindien. Nova Hedwigia 11: 1-73.

Singh, A. (1980). Lichenology in Indian Subcontinent 1966-1977. Economic Botany Information Service, National Botanical Research Institute, Lucknow, 1-112pp.

Singh, K.P. \& G.P. Sinha (2010). Indian Lichens An Annotated Checklist. Botanical Survey of India. Shiva Offset Press, 571pp.
Sipman, H.J.M. (1994). Foliicolous lichens on plastic tape. Lichenologist 26: 311-312.

Tetali, P., S. Tetali, B.G. Kulkarni, P.V. Prasanna, P. Lakshminarasimhan, M. Lale, M.S. Kumbhojkar, D.K. Kulkarni \& A.P. Jagtap (2000). Endemic Plants of India: A Status Report of Maharashtra State. Naoroji Godrej Centre for Plant Research, Shirwal, 87pp.

Watve, A. (2013). Status review of rocky plateaus in the NW Ghats and Konkan region of Maharashtra with recommendations for conservation planning. Journal of Threatened Taxa 5(5): 3935-3962; http://dx.doi.org/10.11609/JoTT.o3372.3935-62

Widdowson, M. (1997). Tertiary palaeosurfaces of the SW Deccan, western India: implication for passive margin uplift, pp. 221-248. In: Widdowson M. (ed.). Palaeosurfaces: Recognition, Reconstruction and Palaeoenvironmental Interpretation. Geological Society Special Publication - 120.

Widdowson, M. \& K.G. Cox (1996). Uplift and erosional history of the deccan traps India: evidence from laterites and drainage patterns of the Western Ghats and Konkan coast. Earth and Planetary Science Letters 137: 57-69. 